\title{
ARTIFICIAL NEURAL NETWORK BASED ONLINE SENSOR CALIBRATION AND COMPENSATION
}

\author{
Shakeb A. Khan ${ }^{1)}$, Tarikul Islam ${ }^{2)}$, Gulshan ${ }^{3)}$ \\ ${ }^{1)}$ Department of Data Communication \& Networking, College of Computer Engineering, \\ King Khalid University, Abha, Kingdom of Saudi Arabia. e-mail: khanshakeb@yahoo.com \\ ${ }^{2)}$ Department of Electrical Engineering, Jamia Millia Islamia (A Central University), \\ New Delhi-110025, INDIA. e-mail: tariq940@rediffmail.com \\ ${ }^{3)}$ Electrical Engineering Section, University Polytechnic, Jamia Millia Islamia (A Central University), \\ New Delhi-110025, INDIA. e-mail: gulshanhusain@gmail.com
}

\begin{abstract}
This paper presents an artificial neural network (ANN) based generalized online method for sensor response linearization and calibration. Inverse modeling technique is used for sensor response linearization. Multilayer ANN is used for inverse modeling of sensor. The inverse model based technique automatically compensates the associated nonlinearity and estimates the measurand. The scheme is coded in MATLAB® for offline training and for online measurement and successfully implemented using NI PCI-6221 Data Acquisition (DAQ) card and LabVIEW® software. Manufacturing tolerances, environmental effects, and performance drifts due to aging bring up a need for frequent calibration, this ANN based inverse modeling technique provides greater flexibility and accuracy under such conditions.
\end{abstract}

Keywords: Artificial neural networks, calibration, compensation, sensor, inverse modeling, nonlinearity.

\section{INTRODUCTION}

Sensors and transducers are widely used for the quick and precise measurement of many parameters. The nonlinearity associated with these sensors often creates several difficulties in direct digital readout and in control applications. Manufacturing tolerances, environmental effects and performance drifts due to aging bring up a need for frequent recalibration. When the characteristic of a sensor is linear, the output signal is carried out upon the displayed instrument panel. This operation might be a simple scaling, removing dc level and amplification. If the sensor output is nonlinear, it will produce a whole assortment of new problems. In this case, the sensor output requires corrections for input/output nonlinearities. To solve the problem of linearizing a sensor, there are in general two methods, the first one requires nonlinear analog circuit and the second uses numerical methods that are computed by microprocessor or personal computer. This paper presents a computer based online measurement technique which estimates the measurand by neutralizing the sensor nonlinearity.

To convert the nonlinear response of a sensor into a linearized form, several digital techniques, like Read Only Memory (ROM) based look-up-table technique, Analog to Digital Converter (ADC) based nonlinear coding scheme $[1,2]$ and ANN based techniques [3-5] are used. The classical statistical regression modeling is also used to determine the polynomial approximation to the characteristics of the sensor $[6,7]$.

In case of any recalibration, in the former two techniques, either full rewriting of the lookup table and/or modification of the decoding function is needed. The latter two techniques require only the coefficients of the interpolating polynomial to be evaluated, which requires only few new calibration data points. The order of the interpolating polynomial and number of recalibration points needed for a particular extent of the sensor nonlinearity for a given desired Root Mean Square (RMS) error are important required inputs for these latter techniques.

In the ANN based sensor linearization technique reported in [3] an ANN based inverse modeling technique has been used to find the best-fit curve for sensor characteristics to match some given calibration points. The inverse model can be evolved by an iterative learning procedure in such a way that combined transfer function of sensor and its inverse model becomes unity. As a result, the applied measurand can be accurately estimated at all points 
in the range, at the output of the inverse model, irrespective of type of non-linearity of the sensor.

In this paper a generalized online ANN based method is proposed for sensor linearization and compensation. Instead of ADALINE based ANN a multi layer ANN (MLNN) is used to tackle the high nonlinearity cases. The ANN based sensor linearization technique is implemented using NI PCI-6221 DAQ card and LabVIEW 8.0 software. A NTC Thermistor, having 51\% nonlinearity, is used to test the performance of the method. Network training is done off line on MATLAB ${ }^{\circledR}$ using backpropagation algorithm. Training and testing data is acquired using using DAQ card. Weights of the trained network are used for online measurement. Virtual Instruments (VI) setup is developed as front end using LabVIEW ${ }^{\circledR}$ for online signal conditioning and measurement of temperature using Thermistor. Complete on line measurement setup is developed using DAQ card, LabVIEW $^{\circledR}$ and MATLAB ${ }^{\circledR}$ software. The results obtained from the setup have maximum deviation of $\pm 0.95 \%$ of the true value.

\section{THERMISTOR MODEL}

The characteristic equation for Thermistor is given by

$$
R_{T_{1}}=R_{T_{0}} e^{-\beta / T_{1}}
$$

where, $R_{T 1}=$ Thermistor resistance at $\mathrm{T}_{1}{ }^{0} \mathrm{~K}, R_{T 0}$ $=$ Thermistor resistance at reference temperature $(0$ $\left.{ }^{0} \mathrm{C}\right), \beta=$ is a constant depending upon the material of the Thermistor, typically 3500 to $4500{ }^{0} \mathrm{~K}$.

The inverse characteristic equation derived from the above equation is given by

$$
T_{1}=\frac{\beta}{\ln \left(\frac{R_{T_{0}}}{R_{T_{1}}}\right)}
$$

The following power series expansion may represent the inverse response characteristics for thermistor:

$$
T_{1}=\sum_{i=0}^{n} a_{i} R_{T_{1}}^{i}
$$

\section{SENSOR INVERSE MODELING}

Inverse modeling based sensor linearization scheme is illustrated in Fig. 1. Here a multilayer neural network (MLNN) is used simulate a nonlinear inverse function of a Thermistor. There are several ways that multilayer neural network can have their connection weights adjusted to learn mappings. Here the popular back-propagation (BP) algorithm, which is a generalization of the LMS algorithm, is used to train the proposed network. The details of the BP algorithm may be seen in $[8,9]$.

Two-layer MLP architecture with single neuron in the output layer is chosen for inverse response characteristics modeling of considered neuron. The proposed ANN is simulated in MATLAB $^{\circledR}$ environment using neural network toolbox functions. For hidden layer logsig transfer function and for output layer purelin transfer function has been selected. The trainlm function is used to train the network.

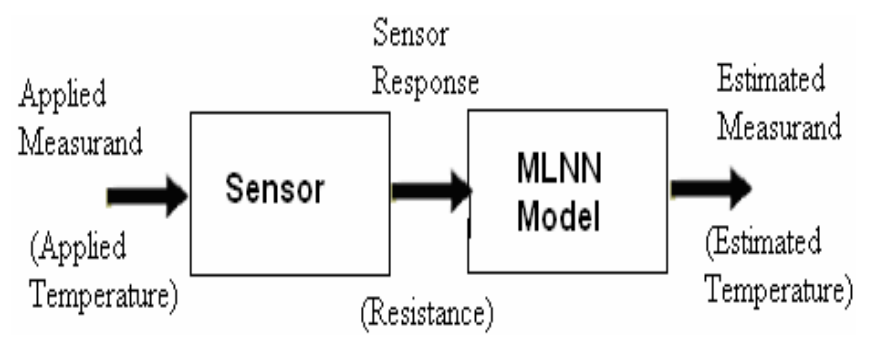

Fig. 1 - Schematic of Sensor Linearization using Inverse Modeling Technique

Extensive simulation studies were carried out with different number of hidden layer neurons and learning rates in order to find an optimum inverse model of Thermistor. The weights and bias values of the network at the specific learning rate and the number of hidden layer neurons for which the training and testing characteristics overlaps are frozen and at that point simulation is stopped. These stored weights and bias are than loaded to the network during online testing of the developed model. All simulation work is done in MATLAB ${ }^{\circledR}$.

\section{IMPLEMENTATION}

The ANN model is trained and tested offline using MATLAB $^{\circledR}$ to estimate the important modeling parameters for varying degree of sensor nonlinearities. Then with the help of these estimated parameters, scheme is implemented with the help of NI PCI-6221 DAQ card and LabVIEW ${ }^{\circledR} 8.0$ software.

\section{A. Training of ANN Model}

ANN training is carried out offline. For training and testing real-time data is acquired in the range of $5-68{ }^{\circ} \mathrm{C}$. Fig. 2 shows the block diagram of the experimental setup used for acquiring the training and testing data points. 


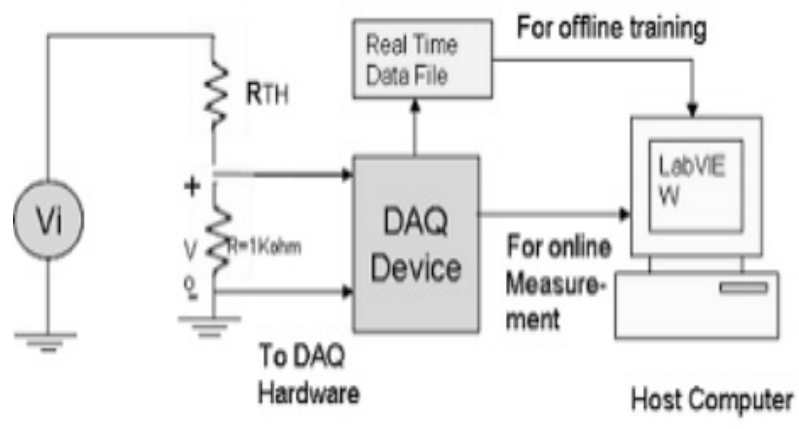

Fig. 2 - Experimental Setup for Online Measurement

In this figure the data acquisition device measures the voltage across a resistor of $1 \mathrm{~K} \Omega$. With the help of (4), Thermistor resistance is computed at different values of temperature, from the measured voltages.

$$
R_{T h}=\frac{V_{i}-V_{0}}{V_{0}} * R
$$

where, Rth =Thermistor resistance; $\mathrm{R}=$ Series Resistance(1Kohm); Vi = Applied DC Voltage (5 volts); $\mathrm{V} 0=$ Voltage across $1 \mathrm{~K} \Omega$ resistance.

The network has been trained with 5, 10 and 15 hidden layer neurons. The selected values of learning rates are $0.01,0.05,0.1,0.15,0.2,0.25,0.3$, $0.35,0.4,0.45$ and 0.5 . At the first step the number of hidden layer neurons is chosen as 5 and the network is trained by using chosen values of different learning rates and the training and testing characteristics are plotted. The same process is repeated for 10 and 15 hidden layer neurons. when we get overlapping training and testing characteristic we stops the simulation and the weights and bias for the corresponding hidden layer neurons and learning rates are retrieved. These retrieved weights and bias values are then used in the final model.

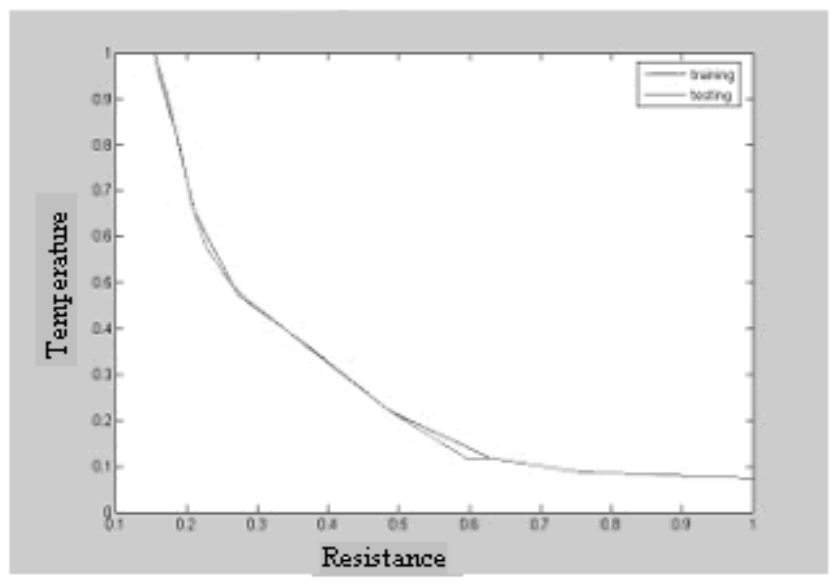

Fig. 3 - Training and Testing Characteristic of ANN based Inverse Model

From the simulation it was found that 1-5-1 MLP structure and the learning rate of 0.05 provide optimum performance. Fig. 3 shows the training and testing characteristic for the same MLP structure and learning rate. From this figure it was find out that the training and testing characteristics of the MLNN model are coinciding at almost all the points. Therefore the weights and biases corresponding to this model are used in the final MLNN model that has been used for on-line testing.

\section{B. On-line testing}

Finally the trained network is used for online measurement. The developed MLNN model is tested with real time data from a $10 \mathrm{~K} \Omega$ Thermistor. Figs. 4 and 5 shows the LabVIEW $^{\circledR}$ block diagram and resultant front panel respectively for online version of the ANN based sensor calibration and compensation scheme.

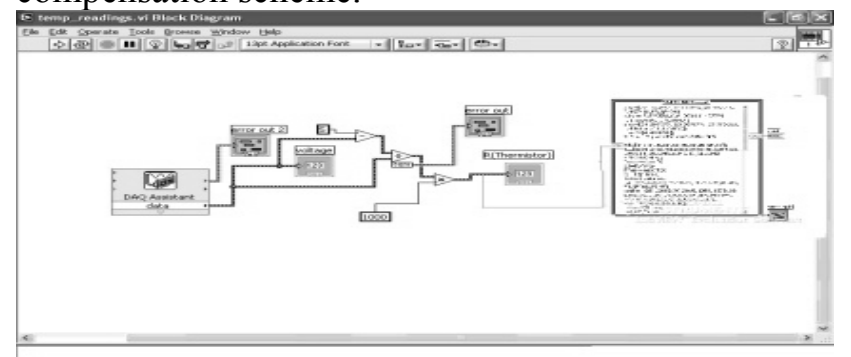

Fig. 4 - Block Diagram for Testing of ANN based Sensor calibratin Scheme

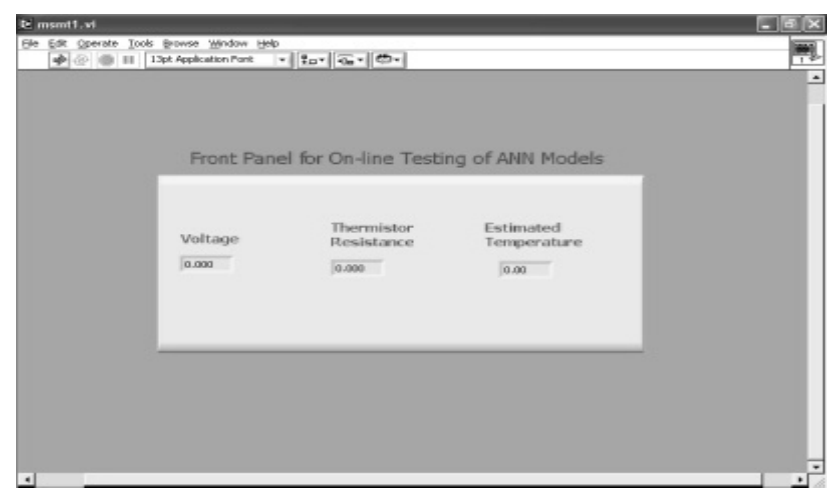

Fig. 5 Front End of the Implemented Scheme

In the block diagram Matlab Script node is used for writing the MATLAB ${ }^{\circledR}$ codes. The resistance of Thermistor (Rth) at particular temperature computed by LabVIEW ${ }^{\circledR}$, using equation (4), is used as input to Matlab script node and from the output of MATLAB $^{\circledR}$ script node we get the corresponding estimated value of the temperature. The front panel of the online testing VI has three online indicators; first for the voltage across the known series resistance, second for thermistor resistance and third for corresponding estimated temperature.

The developed MLNN model is tested with 11 different testing points. Average value of the percentage error between actual temperature and the temperature estimated by the developed MLNN 
model is $\pm 0.95 \%$.

\section{CONCLUSION}

Paper presents implementation of a computer based online sensor calibration and compensation scheme. The scheme is implemented using NI PCI6221 DAQ card, LabVIEW ${ }^{\circledR}$ and MATLAB $^{\circledR}$ software. The calibration scheme is based upon the ANN based inverse modeling of the sensor, which automatically neutralizes the nonlinearity present in the sensor response and estimates the measurand. Extensive simulation study has been carried out in order to find out the optimum number of hidden layer neurons and the value of learning rates for which training and testing characteristics overlaps the actual response characteristics of the considered sensor over the selected span.. We have found that a small 1-5-1 MLNN (one input neuron, five hidden ones and one output) with sigmoid (non-linear) activation function for the hidden neurons and linear for the output neuron, is enough for the purpose. The developed models is successfully tested with a Thermistor having 51\% nonlinearity over the considered span. The achieved accuracy is $\pm 0.95 \%$.

Under changing environmental conditions, for correct readout, the ANN is to be retrained which requires few new data points and small number of epochs to get new set of weights. These new set of obtained weights are entered into MATLAB ${ }^{\circledR}$ script file very easily. Thus this ANN based modeling technique provides greater flexibility and accuracy in changing environment.

\section{REFERENCES}

[1] H. Matsumoto, H. Shimizu, and K. Watanabe, "A switched capacitor charge balancing analogto-digital converters and its application to capacitance measurement," IEEE Trans. Instrum. Meas., vol. IM-36, pp. 873-878, Dec. 1987.

[2] M. Yamada, T. Takebayashi, S.-I. Notoyama, and K. Watanabe, "A switched capacitor interface for capacitive pressure sensors," IEEE Trans. Instrum. Meas., vol. 41, pp. 81-86, Feb. 1992

[3] J. C. Patra, G. Panda and R. Baliarsingh, "Artificial Neural Network based Nonlinearity Estimation of Pressure Sensors", IEEE Trans. Instrum.and Meas., vol. 43, No. 6, pp. 874-881, Dec. 1994

[4] Khan Shakeb A., Shahani D. T., and Agarwala A. K., "Artificial Neural Network (ANN) Based Nonlinearity Estimation of Thermistor", Proc. Of $24^{\text {th }}$ National Sys. Conf. (NSC 2000), ISRO Satellite Cetre, Banglore, pp. 296-302, Dec. 2000 .
[5] Khan Shakeb A., Shahani D. T., Agarwala A. K., "Sensors Calibration and Compensation using Artificial Neural Network", ISA Transactions 42, pp.337-352, 2003.

[6] Pereira Dias J. M., Girao Silva P.M.B., and Postolache Octavian, "Fitting Transducer Characteristics to measured Data", IEEE Instrum. And Meas. Magazine, pp. 26-39, Dec 2001.

[7] Allipi C., Piuri V., "Regression Type Neural network for System Identification", IEEE Instrum. And Meas. Conf. 1995, IMTC/95. Proceedings "Intregrating Intelligent Instrumentation and Control", pp. 311-316, 2426, April 1995.

[8] Haykin, S., Neural Networks, a Comprehensive Foundation, Prentice-Hall, Eaglewood Cliffs, NJ, 1994.

[9] Widraw B. and Lehr M. H. , "30 years of Adaptive Neural Networks: Perceptrons, Madaline, and Backpropagation" IEEE proc. Instrum.and Meas, vol.- 78, PP.1415-1442, Sept. 1990.

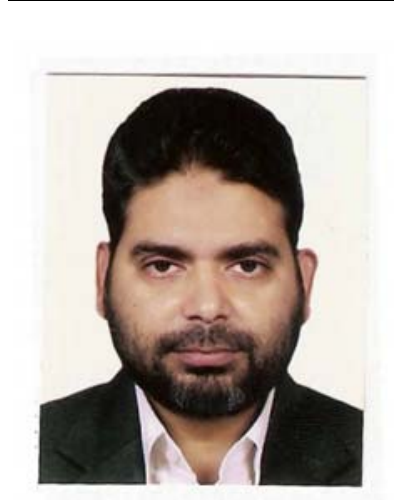

Shakeb A. Khan obtained his B.Sc. Engg. degree in Electrical Engineering and M.SC. Engg. degree in Instrumentation \& Control from the Aligarh Muslim University, India in 1992 and 1994 respectively. He received the Ph.D. degree in the field of Instrumentation from Indian Institute of Technology, Delhi, India in 2005. He joined the Jamia Millia Islamia (A Central University), New Delhi, India, in 1995 and has been there ever since. Presently, he is on leave from Jamia Millia Islamia and woking as Assoc. Prof. in Department of Data Communication \& Networking, College of Computer Engineering, King Khalid University, Abha, KSA His research and teaching interests include electronics instrumentation, artificial neural networks applications, and FPGA based design.

Tarikul Islam obtained his B.Sc. Engg. degree in Electrical Engineering and M.Sc. Engg. degree in Instrumentation \& Control from the Aligarh Muslim University, India. He received the Ph.D. degree in the field of Instrumentation from Indian Jadavpur University, Kolkata, India in 2007. He joined the Jamia Millia Islamia (A Central University), New Delhi, India, in 1998 and has been there ever since. Presently, he is Reader in Department of Electrical Engineering, Jamia Millia Islamia (A Central University), New Delhi, India. His research and 
teaching interests include Capacitive Sensor, Instrumentation, Softcomputing Techniques for signal processing, Microcontroller based signal conditioning.

Gulshan obtained her B.Tech. degree in Electrical Engineering and M.Tech. degree in Electrical Power System Management from the Jamia Millia Islamia (A Central University), New Delhi, India. Presently, She is working as guest faculty, in University Polytechnic, Jamia Millia Islamia (A Central University), New Delhi, India. Her research and teaching interests include Power Systems, and Application of Softcomputing Techniques in Power System. 